University of South Florida

DIGITAL COMMONS

Digital Commons @ University of

@ UNIVERSITY OF SOUTH FLORIDA

South Florida

School of Geosciences Faculty and Staff

Publications

School of Geosciences

3-1990

\title{
Some Tests of Wet Tropospheric Calibration for the CASA Uno Global Positioning System Experiment
}

Timothy H. Dixon

California Institute of Technology, thd@usf.edu

S. Kornreich Wolf

California Institute of Technology

Follow this and additional works at: https://digitalcommons.usf.edu/geo_facpub

Part of the Earth Sciences Commons

\section{Scholar Commons Citation}

Dixon, Timothy H. and Wolf, S. Kornreich, "Some Tests of Wet Tropospheric Calibration for the CASA Uno Global Positioning System Experiment" (1990). School of Geosciences Faculty and Staff Publications. 511.

https://digitalcommons.usf.edu/geo_facpub/511

This Article is brought to you for free and open access by the School of Geosciences at Digital Commons @ University of South Florida. It has been accepted for inclusion in School of Geosciences Faculty and Staff Publications by an authorized administrator of Digital Commons @ University of South Florida. For more information, please contact digitalcommons@usf.edu. 


\title{
SOME TESTS OF WET TROPOSPHERIC CALIBRATION FOR THE CASA UNO GLOBAL POSITIONING SYSTEM EXPERIMENT
}

\author{
T. H. Dixon and S. Kornreich Wolf
}

Jet Propulsion Laboratory, California Institute of Technology

\begin{abstract}
Wet tropospheric path delay can be a major error source for Global Positioning System (GPS) geodetic experiments. We investigate strategies for minimizing this error using data from CASA Uno, the first major GPS experiment in Central and South America, where wet path delays may be both high and variable. We compared wet path delay calibration using water vapor radiometers (WVRs) and residual delay estimation, with strategies where the entire wet path delay is estimated stochastically without prior calibration, using data from a $270 \mathrm{~km}$ test baseline in Costa Rica. Both approaches yield centimeter-level baseline repeatability and similar tropospheric estimates, suggesting that WVR calibration is not critical for obtaining high precision results with GPS in the CASA region.
\end{abstract}

\section{Introduction}

Uncertainties in the correction for variable wet tropospheric path delay can be a major error source for Global Positioning System (GPS) geodesy. Depending on weather conditions and unmodelled satellite orbit effects, wet path delays may be the dominant error for baselines (station separations) longer than about $10-20 \mathrm{~km}$ and shorter than about $500 \mathrm{~km}$, a critical range for many geological studies. We investigate strategies for minimizing troposphere-related errors with data from CASA Uno, the first GPS experiment in Central and South America [Kellogg et al., 1989]. Wé compare several calibration and modeling strategies on a test baseline where a variety of instrumentation for tropospheric calibration was deployed. Tropospheric wet path delays in this region were both high (often exceeding $20 \mathrm{~cm}$ at zenith) and variable (typically 3-6 cm variation over 8 hours) and hence a significant error source in the GPS baseline estimates.

Previous studies [Tralli et al., 1988; Dixon et al., 1990] indicate that wet tropospheric calibration for GPS based on simple atmospheric models and surface measurements of temperature and relative humidity may not be satisfactory. Better results are often obtained with water vapor radiometer (WVR) calibration and estimation of a residual correction, or through estimation of the entire wet delay without prior calibration, relying on the geometric strength of the GPS observables and the known elevation angle dependence of the wet path delay. Estimation is improved if a stochastic model of the temporal variation of delay or its residual after calibration is also used. In this paper, we model the temporal variation of the zenith wet path delay, or its residual after calibration, as a random walk stochastic process, specified by the process noise rate, $\alpha$, with a typical range of 0.2-2.0 $\mathrm{cm} / \mathrm{hhr}$ [Dixon et al., 1990]. If estimation of the entire wet delay gives results equivalent to WVR calibration, the former method is preferable due to the cost of WVR deployment. This is important for CASA Uno because instrument availability limited WVR calibration to a few sites in the CASA region (including the two studied here) and several U.S. sites.

Copyright 1990 by the American Geophysical Union.

Paper number 90GL00421.

0094-8276/90/90GL-00421\$03.00

\section{The CASA Uno Experiment and Data Analysis}

The CASA Uno experiment is described by Kellogg et al. [1989]. Our test baseline for tropospheric calibration is in Costa Rica between Limon and Liberia, $270 \mathrm{~km}$ in length. Limon is located near sea level on the Caribbean coast, while Liberia is located at an elevation of $215 \mathrm{~m}$, about $20 \mathrm{~km}$ east of the Pacific coast. All sites were occupied by TI-4100 GPS receivers that recorded data at 30 second intervals, further compressed in our analysis to 6 minute points. Surface meteorological equipment for automatic recording at 5 minute intervals of temperature $\left( \pm 0.1^{\circ} \mathrm{C}\right)$, pressure $( \pm 0.3 \mathrm{mb})$ and relative humidity ( $\pm 2 \%$ for relative humidity $0-80 \%, \pm 3 \%$ for relative humidity $80-100 \%$ ) was available at Liberia. Conventional meteorological equipment with manual data recording at 60 minute intervals was deployed at Limon, with corresponding precisions approximately $\pm 0.5^{\circ} \mathrm{C}, \pm 1.0 \mathrm{mb}$ and $\pm 5 \%$ in relative humidity. Liberia was equipped with a solar hygrometer, and both Limon and Liberia had state of the art "D-Series" WVRs. The two channel D-Series are modeled closely after the high precision 3-channel "J-Series" radiometer, designed to yield estimates of the zenith wet path delay with a precision better than $1.0 \mathrm{~cm}$ [Janssen, 1985]. With fewer channels and simplified temperature stabilization circuitry, D-Series instruments are lighter in weight and operate with less power relative to the J-Series, enabling operation in remote areas with only slight degradation in precision. Some constraints on the precision and accuracy of the data acquired by these instruments during CASA Uno are discussed below. The WVRs were operated in the "tip curve" mode, with atmospheric brightness temperature measurements taken at several predetermined elevation angles on alternate east-west and north-south azimuths every 3-4 minutes and processed to yield the zenith delay. All the approaches we tested for calibration or estimation of the wet path delay assume that the delay is azimuthally symmetric, i.e., only a zenith delay is determined; delays at other elevations are determined with a mapping function [Lanyi, 1984]. The validity of this assumption will be discussed. The dry delay is obtained by measurement of surface pressure under the assumption of hydrostatic equilibrium. Since the wet and dry mapping functions are essentially identical above $15^{\circ}$ elevation, the cutoff angle used in our analysis, any miscalibration of the dry delay is absorbed by estimation of the zenith wet delay or its residual after calibration.

Our analytical techniques are described in Tralli et al. [1988], Blewitt [1989] and Dixon et al. [1990]. We selected a subset of CASA Uno data based on several criteria to minimize errors other than those associated with wet tropospheric effects. Initial studies indicated that eight stations in Central and northern South America provide an adequate network for local baseline estimation, ensuring resolution of most of the carrier phase cycle ambiguities, a necessary condition for attaining high precision and accuracy [Dong and Bock, 1989; Blewitt, 1989]. Also, we required that GPS data be available from seven globally distributed tracking stations to minimize orbitrelated errors [Kornreich Wolf et al., this issue] and that at least one hour of WVR data be available at both Limon and Liberia (Table 1). Five out of fifteen days satisfied these criteria and were used in our analysis. Tropospheric calibration or estimation techniques were varied only at Limon and Liberia. At all other sites (seven global tracking sites plus 
TABLE 1. WVR data* (hours) acquired at Limon and Liberia, Costa Rica, January 19-26, 1988.

\begin{tabular}{llllll}
\hline Site & 19 & 20 & 21 & 25 & 26 \\
\hline Limon & 2.0 & 1.0 & 3.5 & 8.2 & 8.5 \\
Liberia & 7.2 & 6.5 & 8.0 & 9.0 & 8.5
\end{tabular}

*Acquired with 2-channel (20.7 and $31.4 \mathrm{GHz}) \mathrm{D}$ -

Series water vapor radiometers, $7^{\circ}$ beam width.

the remaining 6 sites in Central and South America), we used a randorn walk troposphere model for the entire zenith wet delay with $\alpha=1.8 \mathrm{~cm} / \mathrm{hhr}$, a value based on initial studies using criteria such as day to day repeatability and resolution of carrier phase cycle ambiguities [Dixon et al., 1990].

\section{Results and Discussion}

Surface meteorological data combined with an atmospheric model [Chao, 1974] did not yield precise calibration of the wet tropospheric path delay, similar to results of previous studies [Tralli et al., 1988; Dixon et al., 1990]. Limited solar hygrometer data likewise did not yield precise calibration, because a large fraction of the GPS data were acquired at night, precluding simultaneous GPS and solar hygrometer observations. Given the large and unpredictable fluctuations observed in the wet tropospheric path delay (see below), simultaneity is a critical requirement for any high precision calibration technique.

We concentrate our discussion on two promising approaches, namely WVR calibration of the zenith wet path delay (with or without estimation of a residual delay), and estimation of the wet path delay without prior calibration using a random walk model. Repeatability of the Limon-Liberia baseline, defined as the root mean square (rms) scatter of independent daily baseline estimates about the mean, is shown in Figure 1 for various tropospheric treatments using these two

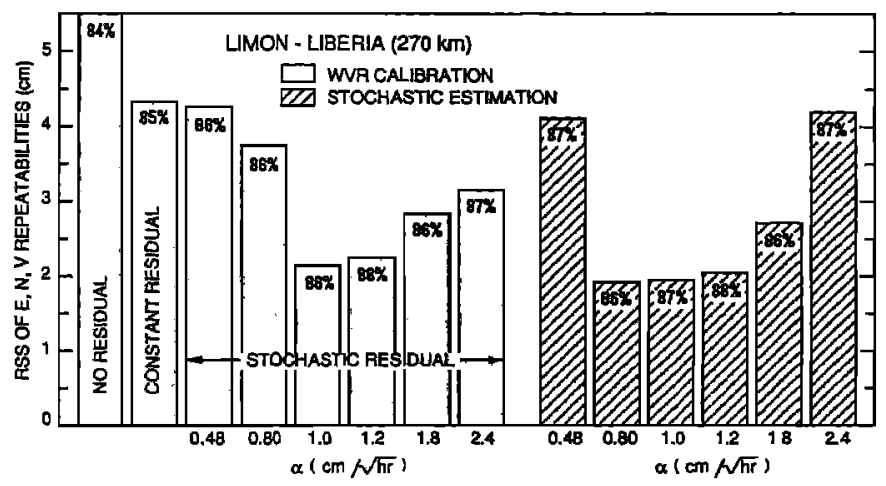

Fig. 1. Repeatability of 5 independent daily estimates for Limon-Liberia baseline $(270 \mathrm{~km})$ for various wet troposphere treatments. WVR calibration (left side) involves (left to right) no estimation of residual error, estimation of constant residual error, and estimation of random walk model of residual error specified by process noise rate, $\alpha(\mathrm{cm} / \mathrm{Vhr})$. Treatments without prior wet calibration (right side) use random walk models for entire wet path delay, also specified by $\alpha$ (same units). From left to right, wet path delay or its residual after calibration is allowed to vary by progressively greater amounts over the observation period. Percentages at top of each bar indicate number of carrier phase biases in the local CASA network resolved for each treatment. overall approaches. With WVR calibration, the worst repeatability is obtained when no residual error is estimated; optimum repeatability with WVR calibration involves estimation of a random walk residual error with $\alpha=1.0$ $\mathrm{cm} / \sqrt{\mathrm{hr}}$, allowing the calibration error to vary up to $2.8 \mathrm{~cm}$ over the 8 hour view period. The WVR calibration scheme that allows the greatest number $(-88 \%)$ of cycle ambiguities to be resolved within the 8 site region in Central and northem South America is very similar, with $\alpha=1.2 \mathrm{~cm} / \mathrm{hhr}$ (up to 3.4 $\mathrm{cm}$ variation in 8 hours). When the entire wet delay is estimated without prior calibration, the best repeatability is obtained with $\alpha=0.8 \mathrm{~cm} / \sqrt{\mathrm{hr}}$, while the maximum number of resolved cycle ambiguities is obtained with $\alpha=1.2 \mathrm{~cm} / \mathrm{hh}$. These values of $\alpha$ are surprisingly close to the optimum model identified for an earlier experiment in the northern Caribbean [Dixon et al., 1990]. They are also within the range obtained by analysis of the time series of zenith wet delays measured on each of the five days by the WVRs; the mean values at Limon and Liberia are, respectively, $1.9 \mathrm{~cm} / / \mathrm{hr}$ (range 1.2-2.6 $\mathrm{cm} / \sqrt{\mathrm{hr}}$ ) and $1.7 \mathrm{~cm} / \mathrm{hhr}$ (range $0.6-3.8 \mathrm{~cm} / \sqrt{\mathrm{hr}}$ ). Attempts to improve baseline repeatability by devising more complicated random walk troposphere models, optimized by site or day to account for local variations, were unsuccessful; a simple model for the entire region over the time span of the experiment gave results indistinguishable from our best "customized" model.

Note that the best WVR calibration scheme (with stochastic residual estimation) yielded repeatability and number of resolved cycle ambiguities almost identical to the case where the entire wet path delay was estimated stochastically without prior calibration. The effect of these two approaches on actual baseline estimates (a way of evaluating effects on accuracy) is shown in Figure 2. The means of the horizontal baseline
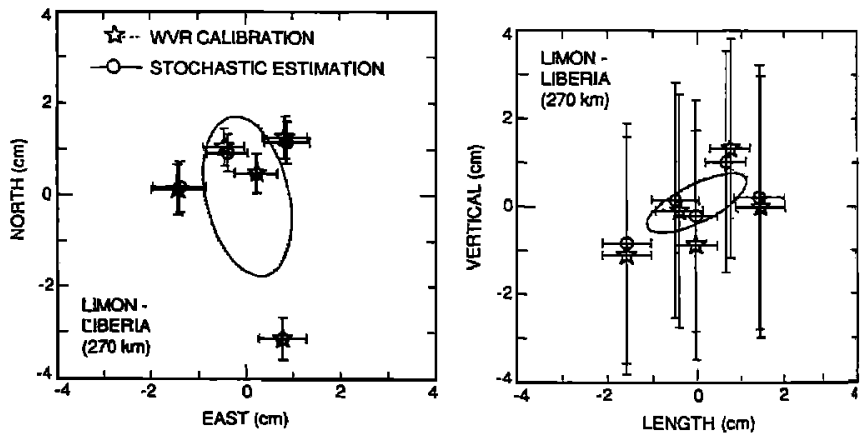

Fig. 2. Five independent vector estimates for the LimonLiberia baseline using two optimum wet troposphere treatments, each based on maximizing number of resolved cycle ambiguities (Figure 1 and text): Calibration with WVR and estimation of stochastic residual error $(\alpha=1.2 \mathrm{~cm} / \mathrm{hbr})$, and stochastic estimation of the entire wet delay (same $\alpha$ ).

estimates from the two techniques agree at the sub-millimeter level, and even the individual daily estimates agree at the 1-2 $\mathrm{mm}$ level, well within the formal errors. However, the means of the two vertical component estimates differ by about $1 \mathrm{~cm}$. Previous observations using very long baseline interferometry (VLBI) suggest that WVR calibration mainly affects the vertical component estimate [Herring, 1986)], and this is apparently true with GPS as well. However, since the formal errors of the vertical estimates exceed $1 \mathrm{~cm}$, the significance of this observation is unclear. We also have no information on which estimate is more accurate.

We draw two conclusions from these observations. First, stochastic estimation techniques for wet tropospheric path delays are sufficiently robust that they can be applied to thoso 
CASA experiment sites lacking WVR calibration, with little degradation in precision or accuracy of the baseline estimates. Our second conclusion is more general, and stems in part from the general agreement between results presented here and previous studies [Tralli et al., 1988; Dixon et al., 1990]. It appears that WVR calibration, of the quality currently available for routine field deployment, under the experiment conditions encountered to date, with current analytical techniques and models, and at current GPS precision levels, yields GPS baseline estimates almost identical to those obtainable with stochastic troposphere estimation techniques.

Why are the GPS baseline estimates not improved by WVR calibration? In part, this reflects the ability of GPS data from a strong network to "self-calibrate" major tropospheric effects using the estimation techniques we have described. GPS data are especially strong if the carrier phase cycle ambiguities are resolved, leading to improved precision and accuracy of horizontal baseline component estimates [Blewitt, 1989; Dong and Bock, 1989]. Our 8 station regional CASA network provided a mix of baseline lengths that allowed geometric resolution of most $(>84 \%)$ cycle ambiguities regardless of tropospheric treatment, reducing the possible impact of WVR calibration. This impact may also be diluted because we are comparing cases where 6 out of 8 stations in our regional network lack WVR calibration, versus 8 out of 8 stations. Perhaps WVR deployment at the majority of stations in a network is required before major advantages to calibration can be observed. Note that loss of some WVR data at Limon (January 19-21; Table 1) cannot explain the failure of WVR calibration to improve the baseline estimates, because the esrimates for all 5 days (Figure 2) are essentially identical for both calibrated (WVR) and non-calibrated (stochastic estimate) cases. Another possibility is that we have not treated residual WVR errors in an optimum manner. While some information suggests that random walk models are appropriate for tropospheric path delays, no such data exist to support their applicability to residual WVR delays. Perhaps with a better model of WVR errors, WVR calibration and estimation of tightly constrained residual errors would be more beneficial.

The accuracy of WVR calibration must be better than $1 \mathrm{~cm}$ in zenith delay in order to improve VLBI baseline estimates [Davis et al., 1985]. For GPS baseline estimates with carrier phase cycle ambiguities resolved, the accuracy requirement for WVR calibration is probably of the order of the inherent precision of the carrier phase measurement, roughly 0.3-0.5 $\mathrm{cm}$ for the GPS receivers used in this experiment Several lines of reasoning, discussed below, suggest that WVR accuracy under the conditions of the CASA Uno experiment may be worse than $0.5 \mathrm{~cm}$ :

1) Formal errors of the WVR zenith delay estimates during the CASA Uno experiment range from $0.2-0.9 \mathrm{~cm}$ (see point 3 , below). Side by side comparisons of D-Series WVRs occasionally show differences in zenith delay in excess of 1 $\mathrm{cm}$, but $\mathrm{mms}$ differences in these comparisons are generally 0.5 $\mathrm{cm}$ or better.

2) Although formal errors of baseline estimates are smallest when WVR calibration is used without estimating a residual error (i.e., calibration assumed correct), scatter in the baseline estimates is increased (Figure 1). The simplest explanation for this is that an error exists in the WVR calibration with a magnitude that exceeds GPS data noise. When a constant residual error is estimated for WVR calibration, the scatter in the baseline estimates is reduced. The magnitude of the residual error correction may be as high as $4 \mathrm{~cm}$ (point 3 , below). The best stochastic residual error models have $\alpha$ in the range 1.0-1.2 cm/ $/ \mathrm{hr}$ (Figure 1); the magnitude of $\alpha$ probably reflects to a large extent the magnitude of the calibration error, and further suggests temporal variation in that error. An alternate possibility is that another error source with a similar mapping function to the wet path delay, e.g., the dry delay, is present in the data and is being corrected by the estimation process. However, we believe this to be unlikely. Dry delays generally exhibit very low temporal fluctuations. The barometers used to derive the dry delay were well calibrated before and (in the case of Liberia) during the experiment; their calibration errors are also unlikely to exhibit large temporal changes.

3) The zenith wet path delay as a function of time at Limon and Liberia can be estimated from the GPS observables alone, independent of the WVR measurements (Figure 3). Ideally,

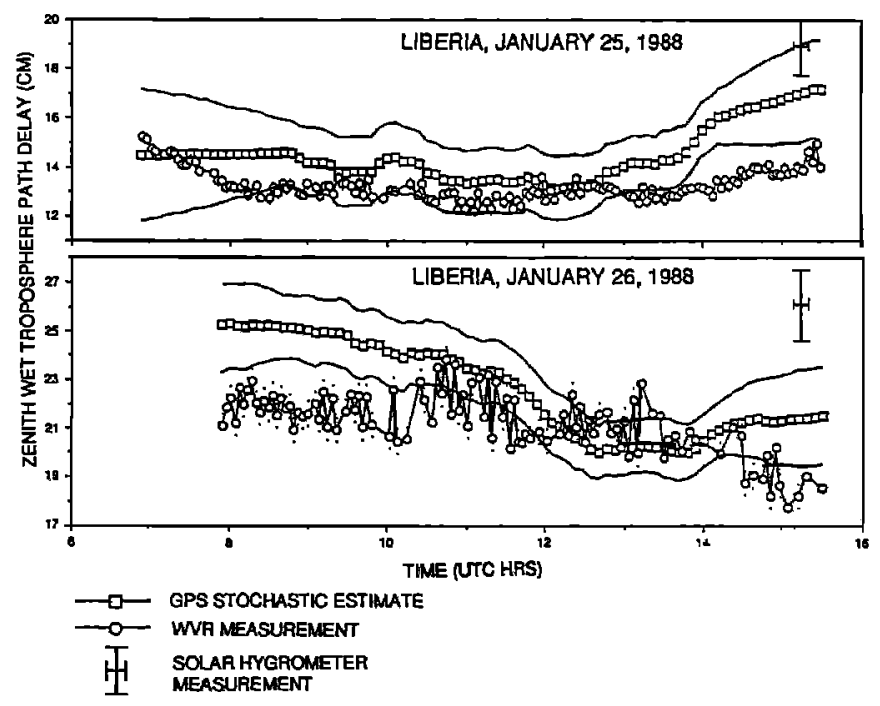

Fig. 3. Comparison of zenith wet tropospheric path delay measured by WVR at Liberia, and estimated solely from the GPS observables without prior calibration, using random walk model ( $\alpha=1.8 \mathrm{~cm} / \sqrt{\mathrm{hr}}$ ), for January 25 (top) and January 26 (bottom). Solid lines outline approximate $\pm 1 \sigma$ formal (data noise) error for GPS estimates, and dots outline mean errors for WVR estimates. Note larger errors for GPS-based estimates at beginning and end of data arcs, and larger errors on WVR estimates for January $26(\sim \pm 0.5 \mathrm{~cm})$ relative to previous day $( - \pm 0.2 \mathrm{~cm})$. Also note large $(\sim 0.5-2.0 \mathrm{~cm})$ differences in adjacent data points for WVR estimates on January 26 , representing alternate north-south and east-west scans at various times (e.g., 10.4-11.6 hours UTC), possibly due to azimuthal asymmetry. Crosses at 15:15 UTC show daily solar hygrometer measurement made near local sunrise.

GPS-based estimates of the zenith wet path delay should agree with WVR-based estimates within the formal errors of the two techniques, or better than about $2 \mathrm{~cm}$. Deviations larger than this can give clues to the nature and causes of systematic errors in the two estimates. Agreement within $1-2 \mathrm{~cm}$ is often obtained, with two exceptions. First, differences between GPS- and WVR-based estimates of zenith wet path delay may exceed $2 \mathrm{~cm}$ at the beginning and end of data arcs (Figure 3), due to weaknesses in the GPS-based estimates, as the number and geometric spread of the satellites is reduced at these times. Occasional differences at the beginning of the data arc may also reflect lack of thermal stabilization in the WVR; logistical considerations at remote sites sometimes preclude the required (one hour or more) warm-up period. More significant, however, are larger differences (up to $4 \mathrm{~cm}$ ) between GPSand WVR-based wet path delay estimates observed on days when large cumulus cloud buildups, rain or heavy condensation occurred. Such effects may degrade the accuracy of the WVR wet path delay estimate [Robinson, 1988]. Figure 3 shows two examples of zenith wet path delay estimates, from two consecutive days in Liberia. On January 25 , the zenith wet delay is relatively low and does not fluctuate 
greatly (3-4 cm over 8 hours) and the WVR measurements have small formal errors (mean and range $0.2 \mathrm{~cm}$ and $0.1-0.4$ $\mathrm{cm}$, respectively). Agreement between the two techniques is good over most of the data arc. In contrast, January 26 has higher mean wet delay and higher fluctuations $(5-6 \mathrm{~cm}$ over 8 hours, with significant short period fluctuations), the WVR measurements have higher formal errors (mean and range 0.5 $\mathrm{cm}$ and 0.2-0.9 cm, respectively), and the two techniques differ by up to $4 \mathrm{~cm}$ in zenith delay even during periods of good satellite geometry. The larger WVR errors on January 26 reflect higher scatter in the tip curve calibration data, almost certainly due to high atmospheric variability and cloud effects during the calibration scans. Since the effects are ubiquitous throughout the 8 hour observation period, it is difficult to edit the calibration data to favor stable periods.

4) Azimuthal asymmetries in the wet path delay may also be important. Inspection of the WVR tip curve data shows that altemate north-south and east-west scans may record different levels of wet delay in a fairly systematic way over short (15-60 minute) time periods (Figure 3 ). Such differences are most easily explained by azimuthal asymmetries; poorly levelled instruments can be ruled out because the effects vary rapidly in time. Asymmetries at the $0.5 \mathrm{~cm}$ or greater level (when mapped to zenith) were observed for periods of 15 minutes or longer on 5 days out of the 10 available observation days. In such cases, the spatial average of the zenith delay parameter obtained from WVR measurements, while approximately correct, may not be the best estimate of the actual line of site delay experienced by a given GPS signal; stochastic estimation of the wet delay may yield a value more representative of the actual delay experienced by the ensemble of GPS signals at that epoch. Line of site WVR calibration to each satellite would be preferable in these conditions. Note, however, that it may be more difficult to maintain accurate WVR calibration with the less frequent tip curves that result from this strategy, an important consideration for portable units like the D-series which may lack sophisticated thermal stabilization. Finally, the spatial asymmetries that we believe are occasionally present also imply rapid temporal fluctuations [Treuhaft and Lanyi, 1987]. It is therefore possible that while the accuracy of the WVR calibration averaged over an hour or mure is at the 0.5 $\mathrm{cm}$ level or better, the short term average (e.g., over the 6 minute period of the GPS compressed data points) is somewhat worse.

In summary, we believe the accuracy of the WVR zenith wet path delay estimates at Limon and Liberia, or the resulting GPS line of site calibration data, was likely worse than the inherent precision of the GPS carrier phase measurements. Consequently, GPS baseline estimates in this experiment were not improved by WVR calibration.

\section{Conclusions}

1. Stochastic estimation of the entire zenith wet path delay without prior calibration, relying solely on the strength of GPS data, yields results that are essentially equivalent to those obtained with calibration by water vapor radiometers (WVRs) for a $270 \mathrm{~km}$ test baseline in Costa Rica within a regional 8 station network. Thus, lack of WVR calibration at the majority of CASA Uno sites should not affect significantly the precision or accuracy of the resulting GPS baseline estimates.

2. The failure of WVR calibration to improve GPS baseline estimates may be due to the presence of: 1) high temporal variability, precluding accurate tip curve calibration of the WVR zenith wet delay estimates, and 2) azimuthal asymmetries, precluding accurate GPS line of site calibration even with accurate WVR zenith delay estimates.
Acknowledgements. We thank all the CASA UNO participants, including Ruth Neilan, Jim Kellogg, the GPS and WVR operators in Costa Rica (K. Clark, R. Pritle, S. Wilkinson, J. M. Protti-Quesada, J. O'Brian and K. Marek), Bruce Gary for help in reducing the solar hygrometer data, and G. Blewitt, C. Edwards, R. Treuhaft, R. King and an anonymous reviewer for helpful comments on the text. This work was conducted at the Jet Propulsion Laboratory, California Institute of Technology, under contract to NASA.

\section{References}

Blewitt, G., Carrier Phase ambiguity resolution for the Global Positioning System applied to geodetic baseline up to 2000 km, I. Geophys Res, 94. 10187-10203, 1989.

Chao, C. C., The troposphere calibration model for Mariner Mars 1971, JPL Technical Report. 32-1587, 61-76, 1974.

Davis, J. L., T. A. Herring, I. I. Shapiro, A. E. E. Rogers and G. Elgered, Geodesy by radio interferometry: effects of atmospheric modelling errors on estimates of baseline length, Radio Science, 20,1593-1607, 1985.

Dixon, T. H., G. Gonzalez, E. Katsigris, S. M. Lichten, First epoch geodetic measurements with the Global Positioning System (GPS) across the northern Caribbean plate boundary, J. Geophys. Res., (In Press), 1990.

Dong, D. and Y. Bock, Global Positioning System network analysis with phase ambiguity resolution applied to crustal deformation studies in California, J. Geophys. Res. 94 . 3949-3966, 1989.

Herring, T. A., Precision of vertical position estimates from very long baseline interferometry, J. Geophys. Res. 21. 9177-9182, 1986.

Janssen, M.A., A new instrument for the determination of radio path delay due to atmospheric water vapor, IEEE Trans, Geoscience and Remote Sensing, GE-23, 485-490, 1985.

Kellogg, J., T. Dixon and R. Neilan, CASA: Central and South America GPS geodesy, Eos Trans. AGU 70, 649 656, 1989.

Kornreich Wolf, S., T. H. Dixon, and J.T. Freymueller, Effect of tracking network configuration on GPS baseline estimates for the CASA Uno experiment, Geophys. Res. Lett. (This issue).

Lanyi, G. E., Tropospheric delay effects in radio interferometry, Telecommunications and Data Acquisition Prog. Rept., 42-78, Jet Propulsion Laboratory, Pasadena, CA, April-June, 152-159, 1984.

Robinson, S.E., The profile algorithm for microwave delay estimation from water vapor radiometer data, Radio Science 23. 401-408, 1988.

Treuhaft, R. N. and G. E. Lanyi, The effects of the dynamic wet troposphere on radio interferometric measurements, Radio Science, 22, 251-265, 1987.

Tralli, D. M., T. H. Dixon, S. Stephens, The effect of wet tropospheric path delays on estimation of geodetic baselines in the Gulf of California using the Global Positioning System, I. Geophys. Res, 23, 6545-6557, 1988.

T. H. Dixon and S. Kornreich Wolf, Division of Earth and Space Sciences, Jet Propulsion Laboratory, Mail Code 183501,California Institute of Technology, Pasadena, CA 91109.

(Received November 6, 1989; Revised February 5, 1990; Accepted February 8, 1990.) 